\title{
Article \\ Electric and Magnetic Design of a Deployable WPT System for Industrial and Defense UAV Applications
}

\author{
Filip Rosu * and Alina Badescu
}

Citation: Rosu, F.; Badescu, A. Electric and Magnetic Design of a Deployable WPT System for Industrial and Defense UAV Applications. Electronics 2021, 10, 2252. https://doi.org/10.3390/ electronics10182252

Academic Editors: Lidia Dobrescu, Wen-Cheng Lai, Kan Yu and Wen Liu

Received: 27 July 2021

Accepted: 6 September 2021

Published: 13 September 2021

Publisher's Note: MDPI stays neutral with regard to jurisdictional claims in published maps and institutional affiliations.

Copyright: (c) 2021 by the authors. Licensee MDPI, Basel, Switzerland. This article is an open access article distributed under the terms and conditions of the Creative Commons Attribution (CC BY) license (https:/ / creativecommons.org/licenses/by/ $4.0 /)$.
Laboratory of Systems with Innovative Antennas, University Politehnica of Bucharest, 061071 Bucharest, Romania; alinabadescu@radio.pub.ro

* Correspondence: filip.rosu@radio.pub.ro

\begin{abstract}
The following paper presents a highly efficient wireless power transfer (WPT) system for unmanned aerial vehicle (UAV) applications. The proposed system is designed as a deployable landing pad, where UAVs can be efficiently charged at distances up to $20 \mathrm{~cm}$, while the UAV is landing. The operation frequency is $50 \mathrm{kHz}$. The current work presents two major contributions that help improve this aspect: a novel RX charging pad geometry and an unconventional design of a low-voltage, high-power DC-AC inverter using discrete MOSFET transistors. Both the pad's geometry and the inverter are designed specifically for UAV applications. The input DC to output AC system efficiency peaks at approximately $95 \%$. The peak efficiency is obtained at power transfers of $625 \mathrm{~W}$. A major difference between the present design and traditionally used state-of-the-art systems is the low DC supply voltage requirement of just $24 \mathrm{~V}$, compared with typical values that range from 50 up to $300 \mathrm{~V}$ at similar output power.
\end{abstract}

Keywords: wireless power transfer; UAV; deployable charging systems; Class-E inverter; circular coils

\section{Introduction}

During recent years, the importance of UAV systems has grown exponentially, both in civil and defense applications. The development and evolution of UAV systems is summarized in [1]. Their popularity is due to the fact that they represent an exceptional candidate for a vast number of applications, such as: video surveillance [2,3], defense [4], geological observations [5], SAR (synthetic aperture radar) [6], etc. In applications in which UAVs are required to function continuously, such as the latest trend of pollination drones, the autonomy of the system becomes crucial [7]. Such systems would greatly benefit from an accessible and remote charging station that can be easily transported and deployed. WPT systems offer several advantages over electrically connected contact-based charging systems [8], among which is a safe operation mode even in wet or dirty environments $[9,10]$ that may be remotely accessed in warzones or hazardous environments or in large areas which are not easily accessible by land vehicles (e.g., forests, mountains, canyons, etc.). The solution proposed in this paper addresses an efficient power transfer in the $200 \mathrm{~W}-1 \mathrm{~kW}$ range [11], at charging distances between 10 and $20 \mathrm{~cm}$. The peak system efficiency of $95 \%$ is obtained at $10 \mathrm{~cm}$ with an output power of $625 \mathrm{~W}$. In prior work, an electric vehicle (EV) charging system is presented [12], with similar efficiencies, and power transfers of over $3.3 \mathrm{~kW}$-when operating at the same height of $10 \mathrm{~cm}$. However, the authors use ferrite bars for both transmitter $(T x)$ and receiver $(R x)$ charging pads to increase coupling. This is not a practical solution for UAVs that have weight limitations (on the $R x$ side). The $R x$ pad proposed in this paper weights less than $300 \mathrm{~g}$, compared to the $15 \mathrm{~kg} T x$ pad. It is obvious that for the UAV applications, the solution using ferrites for $R x$ must be discarded. Eliminating the ferrite bars on the $R x$ side will result in field leakage throughout the UAV and should be taken into consideration when designing the chassis.

The rest of the paper is organized as follows: Section 2 of this paper consists of a brief introduction to WPT basics, where the main concepts and notations used throughout 
the article are explained and defined. Section 3 presents the magnetic design of the $T x$ and $R x$ pads of the prototype, taking into account the optimal trade-off between weight, cost, power transfer, charging area, and efficiency. Further, in Section 4, a VLF (very low frequency) driving system based on Class-E RF power amplifiers (PA) is described. Section 5 provides results obtained from a wide set of experiments. Finally, Section 6 summarizes the paper.

\section{The WPT System}

\subsection{Non-Resonant WPT Systems}

A typical system design of a WPT system is shown in Figure 1. The VLF block converts DC power to a VLF signal, with typical frequencies of tens of $\mathrm{kHz} . L_{T x}$ and $L_{R x}$ are the $T x$ and $R x$ coils' inductance, respectively. $\mathrm{M}$ is the mutual inductance between $L_{T x}$ and $L_{R x}$, $I_{S C}$ is current through $L_{R x}$ when the terminals are shorted, and $V_{o c}$ is the voltage when the coils' terminals are left open. $V_{\text {out }}$ is a DC voltage proportional to the received power and load impedance.
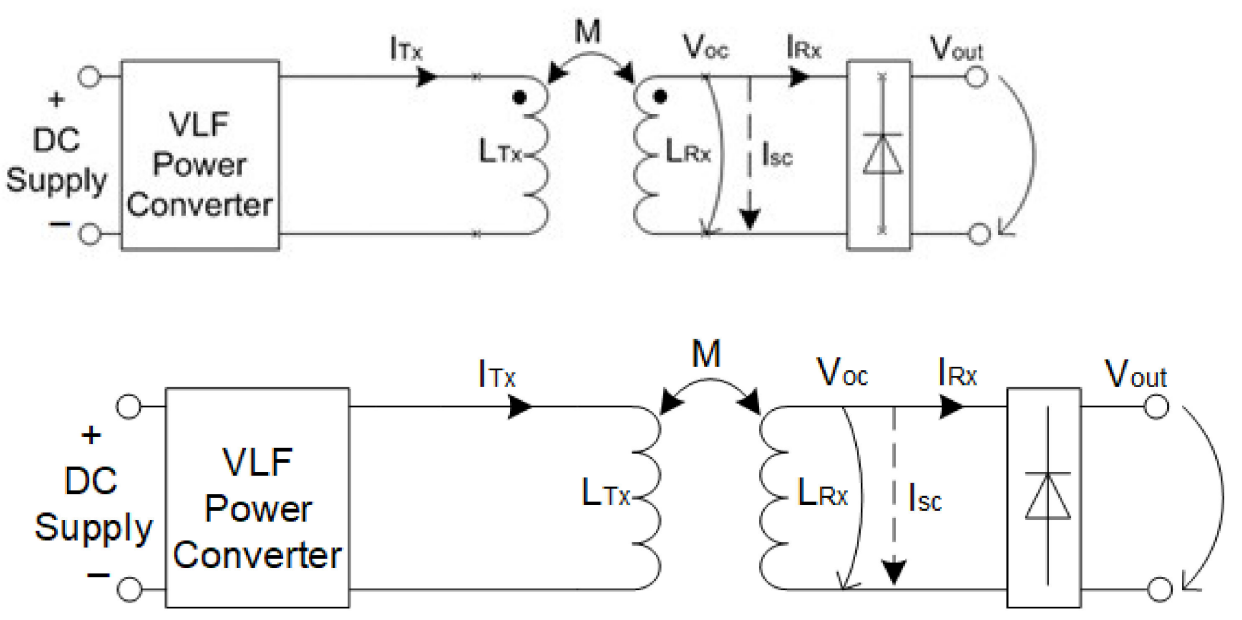

Figure 1. Schematic of an inductive WPT system.

The uncompensated power , $P_{s u}$, is defined as the product between $V_{o c}$, which is affected by core losses, and $I_{s c}$, which depends on copper losses. The maximum active power which can be delivered by an uncompensated system, such as the one presented in Figure 1, is $\frac{P_{s u}}{2}$ [13], where:

$$
P_{s u}=V_{o c} I_{s c}
$$

\subsection{Resonant WPT Systems}

By using resonant techniques, the output power of a WPT system can be expressed as the product between $P_{s u}$ and a factor, $Q$, that depends on the loaded quality factor [14] of the receiver and the compensation topology (which can be series, parallel, or complex). Therefore, when using a resonant LC compensation network, the maximum transferred power is increased by a factor of $2 Q[15]$ :

$$
P_{\text {out }}=P_{s u} Q=V_{T x} I_{T x} k^{2} Q
$$

where $k=M \sqrt{L_{T x} L_{R x}}$ is the coupling factor, and $V_{T x}$ is the output voltage of the $T x$. Equation (2) shows that the output power can be increased if either the coupling factor or the loaded quality factor increases, for a given input power. If the frequency is increased, a higher $Q$ can be obtained [16]; however, copper and switching losses also increase. The configuration is presented in Figure 2, where $R_{T x}$ and $R_{R x}$ represent the resistive losses of the $T x$ and $R x$ coils respectively, and $C_{T x}$ and $C_{R x}$ represent the $T x$ and $R x$ capacitors, respectively. A drawback of the series-series configuration is that maximum 
power transfers can only be obtained at off-resonant frequencies, thus there will always be a trade-off between maximum power output and efficiency.

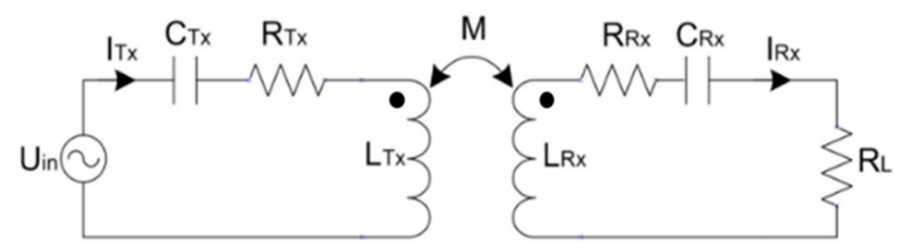

Figure 2. Series-series topology of the resonant WPT system.

It was considered that for the application addressed in this work, the most suited configuration of the two $T x$ and $R x$ LC resonators is series-series. The main reason is that Li-ion batteries have multiple charging stages that require either a constant voltage or a constant current, resulting in a variable load impedance. This makes the series-series configuration a superior choice, because the primary capacitance, $C_{T x}$, is independent of mutual coupling or load impedance, unlike the series-parallel configuration. A more detailed analysis on this topic can be found in $[17,18]$. An important issue is that for a weak coupling between $R x$ and $T x$, large currents are generated at resonance which may permanently damage the transmission system.

Considering that the network is supplied with a harmonic voltage at a frequency equal to the resonant frequency of the two LC series networks, the imaginary component of the impedance vanishes. The solution for the primary and secondary currents, $I_{T x}$ and $I_{R x}$ respectively, becomes:

$$
\begin{aligned}
I_{T x} & =\frac{\left(R_{R x}+R_{L}\right) U_{i n}}{R_{T x}\left(R_{R x}+R_{L}\right)+(\omega M)^{2}} \\
I_{R x} & =\frac{-j \omega M U_{i n}}{R_{T x}\left(R_{R x}+R_{L}\right)+(\omega M)^{2}}
\end{aligned}
$$

Due to the " $-j$ " factor, the waveform at the $R x$ is 90 degrees offset from the $T x$ waveform, and this will be noticed later on in both the Spice simulations and experimental results. Another important observation is that if the system is weakly coupled, so that $\mathrm{M}$ becomes close to 0 , the primary current will become extremely large and can potentially damage the system. This paramount observation will be emphasized in the experimental results section. Alongside power transfer, another important parameter in WPT systems is the transfer efficiency, defined as:

$$
\eta_{T}=\frac{R_{L}\left|I_{R x}\right|^{2}}{U_{i n} I_{T x}}
$$

Therefore, the transfer efficiency can be re-written as:

$$
\eta_{T}=\frac{R_{L}(\omega M)^{2}}{\left[R_{T x} R_{R x}+R_{T x} R_{L}+(\omega M)^{2}\right]\left(R_{R x}+R_{L}\right)}
$$

Figure 3 shows the simulated efficiency as a function of the load resistance. For illustrative purposes, the other parameters were set to: $R_{T x}=0.16 \Omega, R_{R x}=0.03 \Omega$, and $\omega M=3 \Omega$. 


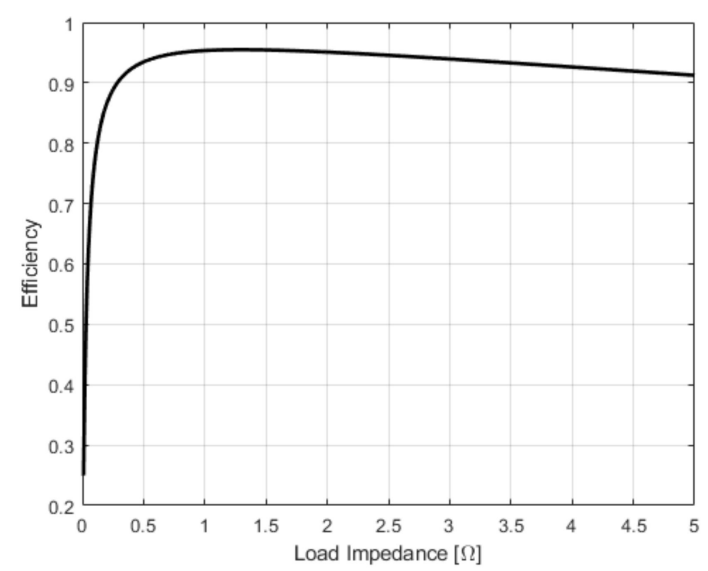

Figure 3. Transfer efficiency as a function of load impedance for $R_{T x}=0.16 \Omega, R_{R x}=0.03 \Omega$, and $\omega M=3 \Omega$.

By solving the null derivative equation $\left(\frac{\partial \eta_{T}}{\partial R_{L}}=0\right)$ and imposing a negative secondorder derivative $\left(\frac{\partial^{2} \eta_{T}}{\partial R_{L}^{2}}<0\right)$, the maximum efficiency requires that:

$$
R_{L}=R_{R x} \sqrt{1+\frac{(\omega M)^{2}}{R_{T x} R_{R x}}}
$$

where $\frac{(\omega M)^{2}}{R_{T x} R_{R x}}=\frac{k^{2} \omega^{2} L_{T x} L_{R x}}{R_{T x} R_{R x}}$ represents the product between the transmitter and receiver quality factors, $Q_{T x}$ and $Q_{R x}$, respectively. By introducing the notation $C=\sqrt{1+k^{2} Q_{T x} Q_{R x}}$, the maximum obtainable power transfer efficiency becomes:

$$
\eta_{\operatorname{Tmax}}=\frac{C-1}{C+1}
$$

Equation (8) proves that by using resonant techniques, the efficiency can be greatly boosted, even when weakly coupled. However, by analyzing Equation (8), one would be inclined to increase the operating frequency of the system to further increase the quality factor, and hence the efficiency. In practice, by increasing the operating frequency, the system losses (i.e., hysteresis losses in the ferrite bars, skin effect losses, switching losses, etc.) also increase. The operating frequency was chosen to be $50 \mathrm{kHz}$ based on the materials and components used.

\section{Charging Pads}

\subsection{Tx Charging Pad Analysis}

In this section, we present a short analysis of both the electrical and magnetic design of the TX pad. The type of LC topology used is series-series. For such a topology, high coupling may cause a double resonance around the initial resonant frequency. This is similar to the effect found in capacitive coupled band-pass filters, which when highly coupled begin to have a double resonance. However, if the resonant peaks are not sharp, this translates into a single larger bandwidth allowing a lower tolerance of the series capacitor value.

Another important aspect that needs to be addressed is the safety standards [19]. An aluminum shielding is required for the coils to isolate the large-field fluctuations from the exterior of the charging pad, even if the aluminum shielding decreases the quality factor of the system. A standard approach in dealing with this problem is to use ferrites, which minimize the flux affected by the shielding. The ferrites are placed below the coils, as shown in Figure 4, and the analysis of the ferrite bar arrangement is discussed in detail in [20]. Another factor that is taken into account by the proposed design is the high voltage 
on the series resonator's $T x$ coil, which can reach up to tens of $\mathrm{kV}$. A well-known solution to this problem is presented in $[8,20]$, where the $T x$ coil is designed as two separate coils connected in parallel, thus reducing the total inductance. The two topologies (i.e., one single coil vs. two coils connected in parallel) are depicted in Figure 4.

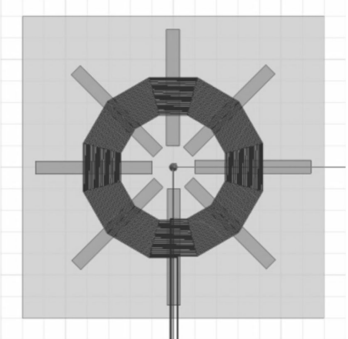

(a)

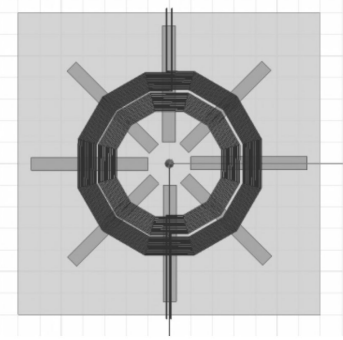

(b)

Figure 4. (a) Single coil design. (b) Double coil design with two concentric coils electrically connected in parallel. A total of 8 ferrite bars are placed below the coils, in a radial configuration.

Figure 5 a presents the electrical configuration, and Figure $5 \mathrm{~b}$ presents the $\mathrm{Z}$ formalism network equivalent. $I_{1}$ and $I_{2}$ are the currents through the inner and exterior coils of the $T x$, respectively.

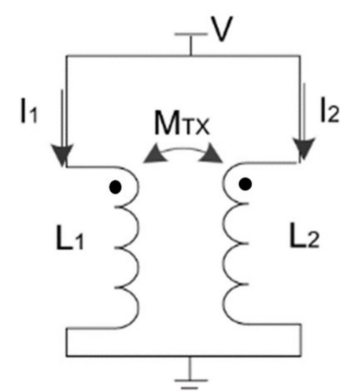

(a)

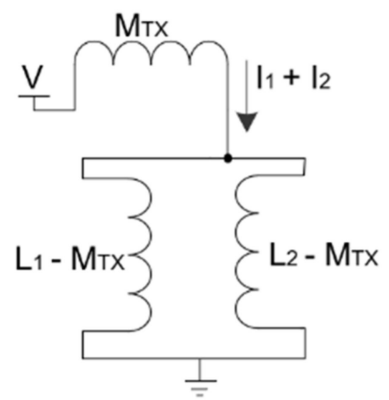

(b)

Figure 5. (a) Electric configuration of the parallel connected Tx coils. (b) The Z formalism network equivalent.

$M_{T X}$ is the mutual coupling between the inner $T x$ coil and the outer $T x$ coil. The total inductance of the parallel in-phase coupled coils is given by:

$$
L_{p}=\frac{L_{1} L_{2}-M_{T X}{ }^{2}}{L_{1}+L_{2}-2 M_{T X}}
$$

Based on the analysis and experimental results obtained in the prior work presented in [21], the diameter of the inner coil was determined to be $D_{1 \text { inner }}=300 \mathrm{~mm}$, such that the entire coil assembly covers $30 \%$ to $60 \%$ of the ferrites' length. The number of turns must be chosen so that the magnetic field in the center of the coil is maximized when supplied by a constant voltage source. The well-known empirical approximation of Harold A. Wheeler for the inductance of a spiral coil [22] is used:

$$
L=\frac{N^{2} A_{e q}^{2}}{30 A_{e q}+11 D_{\text {inner }}}
$$

where $N$ is the number of turns and $A_{e q 1}$ represents an average radius of the coil:

$$
A_{\text {eq }}=\frac{D_{\text {inner }}+w_{s} N}{2}
$$


where $w_{s}$ represents the spacing between the centers of the wires of two adjacent turns. Equations (10) and (11) require that all values be expressed in inches. The currents passing through each inductor, as shown in Figure 5, can be calculated again with the help of the Z formalism:

$$
\begin{aligned}
& I_{1}=\frac{V}{j \omega} \frac{\left(1-\frac{M_{T X}}{L_{2}}\right)}{L_{1}-\frac{M_{T X}{ }^{2}}{L_{2}}} \\
& I_{2}=\frac{V}{j \omega} \frac{\left(1-\frac{M_{T X}}{L_{1}}\right)}{L_{2}-\frac{M_{T X}{ }^{2}}{L_{1}}}
\end{aligned}
$$

In order to optimize the power transfer with respect to the number of turns of each of the two $T x$ coils, information on the variation of the coupling factor is needed. For that, electromagnetic simulations were performed, which served as an input for the following analysis. A finite element method (FEM) electromagnetic solver was used for simulation purposes. Once coupling information was obtained from FEM simulations, numerical simulations were carried out using Equations (12)-(14) to optimize the number of turns required in each coil.

$$
B=\frac{\mu_{0} I_{1} N_{1}}{D_{\text {inner }}+w_{s} N_{1}}+\frac{\mu_{0} I_{2} N_{2}}{D_{\text {inner }}+2 w_{s} N_{1}+w_{s} N_{2}}
$$

The composite B field at the center of the coil, presented in Figure 6, is given by the sum of two elements: the magnetic fields generated by $I_{1}$ through $N_{1}$ turns with an average radius of $\left(D_{\text {inner }}+w_{S} N_{1}\right) / 2$, and the magnetic field generated by $I_{2}$ through $N_{2}$ turns with an average radius of $\left(D_{\text {inner }}+2 w_{s} N_{1}+w_{s} N_{2}\right) / 2$. The ferrite core plays an important role when estimating the currents $I_{1}$ and $I_{2}$, as the arrangement and material of the cores will affect the value of both the mutual inductance and self-inductance. For simplicity, we have ignored the effect of the ferrite bars on the self-inductance, as it only affects the scale of the distribution, and not the shape.

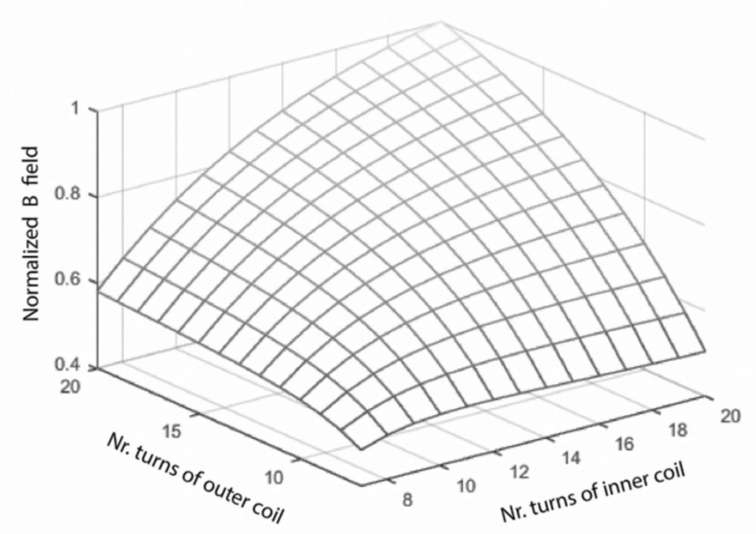

Figure 6. Magnetic flux density, for a constant voltage input, at the center of the pad, as a function of $N_{1}$ (the inner coil number of turns) and $N_{2}$ (the exterior coil number of turns).

The average radius was computed in terms of the distance between two adjacent wires, $w_{s}$, such that for every additional turn, the radius is increased by $w_{s}$. Varying the number of turns also changes the impedance of the coil and, therefore, the input power of the system. During all simulations, the input voltage was maintained constant because the proposed inverter, which is presented in the next section, is modeled as a constant voltage source. There are two normalizations applied to the quantity plotted in Figure 6 and described in Equation (14). The first one is different for each configuration and the field is normalized by the input power (which depends on the currents and inductances). 
The second normalization is a simple scaling with respect to the largest value presented in Figure 6, such that after normalization, the peak is at $0 \mathrm{~dB}$. The normalization was performed since the only quantity of interest was the shape (distribution) and not the absolute values.

\subsection{Rx Charging Pad Analysis}

For the receiver design, all cost, aerodynamics, and weight limitations were considered. This leads to less turns used for the coil and elimination of ferrite bars. Moreover, a large inductance is highly undesired at the receiver end, as it may produce very high voltages. Using capacitors and transistors to step down the voltage can be rather expensive, and the voltage isolation will increase the weight of the UAV. This problem is solved similarly to the $T x$ system, and thus the $R x$ coil is designed from two concentrical, co-planar coils of different sizes and connected electrically in parallel. Unlike the $T x$, where the outer coil and inner coil have similar diameters, the values of the two diameters are significantly different for the $R x$ coil. The FEM simulations presented in Figure 7 were performed for a constant height of $10 \mathrm{~cm}$ across the $R x$ coil. By using a larger coil, the $R x$ pad is less sensitive to positioning errors, while the smaller inner coil is key for high-power transfers. An important design specification is that the diameter of the outer coil of the $R x$ should match the diameter of the outer coil of the $T x$ for an optimal power transfer to size ratio.

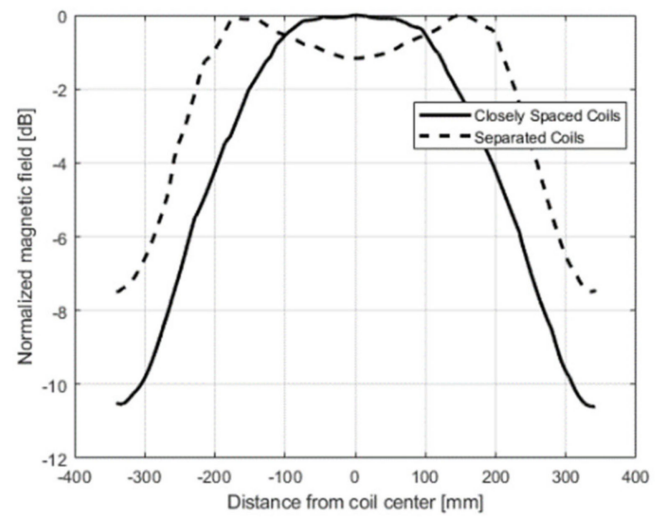

Figure 7. Simulated magnetic field intensity of the $R x$ charging pad (finite element simulation) at a height of $10 \mathrm{~cm}$. The dotted line represents the field distribution when the coils are designed such that both the inner and exterior coil are close together, and the continuous line represents the field distribution when there is a big difference between the inner diameters of the two coils.

A possible optimization method for the size of the inner $R x$ coil is detailed in [23]. The referenced method is based on a priori statistical information on the distribution of the misalignment of the UAV. It further makes use of confidence intervals to optimize the geometry for maximum efficiency. The novelty of the design in the present paper is given by using a small inner coil inside a significantly larger outer coil for optimal weight distribution, power transfer, and misalignment tolerance. The turn/radius ratio of the interior/exterior coils is set to produce equal inductance on the two coils.

Figure 8 presents an aerodynamic and weight-efficient layout example of the $R x$ charging pad placed on a 4-rotor UAV chassis. The dimensions of the coils are determined as follows: As a first step, the exterior $R x$ coil's radius is chosen so it may be placed below the UAV rotors. The interior coil should be attached to the body of the UAV. The number of turns for each coil is chosen such that their inductance is equal. The external $R x$ coil should also not exceed the diameter of the $T x$ coil. 

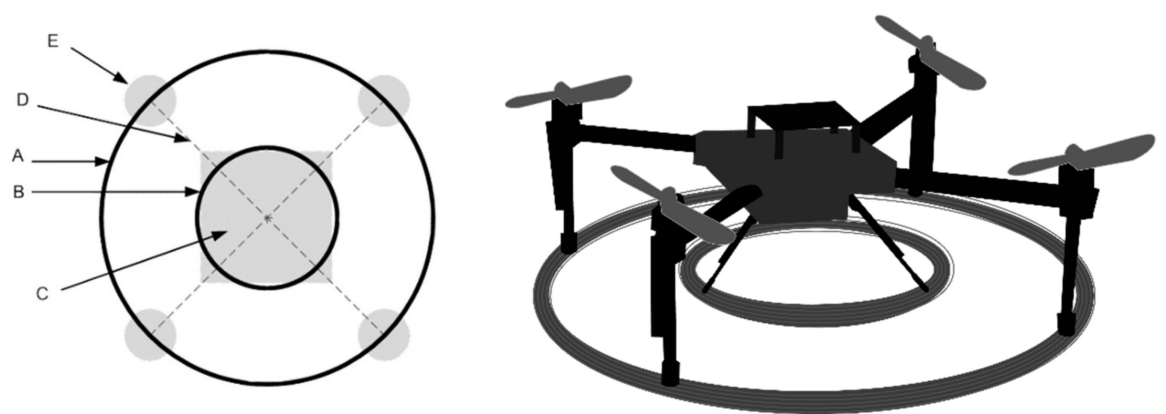

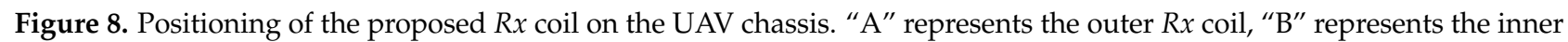
$R x$ coil, " $C$ " represents the UAV chassis, and " $D$ " represents the "wings" of the UAV to which the motors represented by " $E$ " are attached.

\subsection{Design Specifications of the Charging Pad}

Both $T x$ and $R x$ coils have been designed with the same type of Litz wire, having a copper diameter of $1.6 \mathrm{~mm}$ and a copper + isolation diameter of $4 \mathrm{~mm}$. The turn spacing was made as tight as possible. The 8 ferrite bars used were of type N87, with dimensions of $279 \times 30 \times 27 \mathrm{~mm}^{3}$. The aluminum bottom shielding used was $70 \times 70 \mathrm{~cm}^{2}$, and the walls have a $10 \mathrm{~cm}$ height. The thickness of the aluminum shield is $2 \mathrm{~mm}$. The parallel configuration of the two $T x$ coils is shown in Figure $9 a$, and the $T x-R x$ configuration is shown in Figure 9b. Table 1 presents the $T x$ and $R x$ pad specifications.

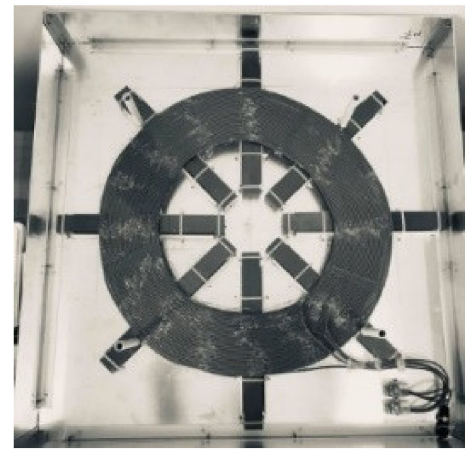

(a)

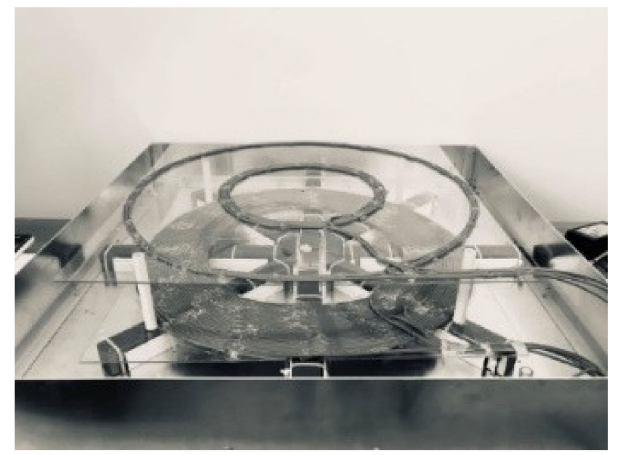

(b)

Figure 9. (a) The $T x$ pad. (b) The $T x-R x$ pads.

Table 1. WPT charging pad specifications.

\begin{tabular}{lccc}
\hline Specifications & Tx Coil & $R x$ Coil & Units \\
\hline Inner coil turns & 14 & 5 & turns \\
\hline Outer coil turns & 14 & 3 & turns \\
\hline Inner coil inner diameter & 300 & 210 & $\mathrm{~mm}$ \\
\hline Outer coil inner diameter & 400 & 475 & $\mathrm{~mm}$ \\
\hline Inner coil self-inductance & 172 & 13.5 & $\mu \mathrm{H}$ \\
\hline Outer coil self-inductance & 218 & 13.8 & $\mu \mathrm{H}$ \\
\hline Inner/Outer mutual inductance & 110 & 1.75 & $\mu \mathrm{H}$ \\
\hline Parallel configuration inductance & 149 & 7.6 & $\mu \mathrm{H}$ \\
\hline Parallel unloaded Q at $50 \mathrm{kHz}$ & 200 & 80 & \\
\hline$T x-R x$ xoupling $(\mathrm{k})$ at $10 \mathrm{~cm}$ & & & \\
\hline
\end{tabular}




\section{Power Inverter Stage}

In order to achieve a high efficiency, a switching class amplifier was used to convert the voltage from the DC power supply to AC current. An ideal Class-E amplifier can be modeled as a switch having a zero voltage drop when conducting and passing zero current when off [24]. Usually in WPT systems, the most common configuration used for the highpower $\mathrm{DC}-\mathrm{AC}$ conversion is the $\mathrm{H}$ bridge configuration $[25,26]$. Typical $\mathrm{H}$ bridge designs use a dual-rail power supply, meaning the total number of batteries must be doubled. Thus, it is not a feasible solution for deployable systems. Another downside is that the output power is generally changed by modifying the gate voltage of the MOSFET transistors, which would require a specific set of transistors or to use PWM [27]. However, PWM would require a modulating signal of approximately $500 \mathrm{kHz}$, which is very impractical in high-power-high-current systems due to the extreme difficulty of phase matching the high-side and low-side transistors. Hitherto, the issue is that the current to voltage ratio for a given output power cannot be easily and robustly controlled by using an $\mathrm{H}$ bridge in this particular application.

An alternative and more efficient approach to $\mathrm{H}$ bridges is to use single-ended Class- $\mathrm{E}$ amplifiers, which are analyzed and presented in detail in [28]. However, these designs lack output power flexibility once the system has been implemented, and are highly influenced by changes of the load impedance.

The design proposed in this paper has a higher theoretical efficiency than $\mathrm{H}$ bridges, and although it cannot offer the same large power transfers, very few UAVs require over $500 \mathrm{~W}$ of instantaneous power. The proposed design for the DC-AC inverter consists of two Class-E amplifiers in a dual-channel configuration, which are matched to the LC compensation network [29] via a transformer-based balun built on a ferrite torus. Figure 10 presents the circuit schematic of the proposed design. The major benefits of the design are that it runs on a single-rail power supply and that higher order compensation networks, such as those in $[30,31]$, are not required, since impedance matching is carried out by changing the turn ratio of the balun transformer.

Furthermore, the four power MOSFETs (two in parallel for each phase) are operating in Class-E, either fully ON or fully OFF, thus dissipating very little power and requiring only a relatively small heat sink. Nonetheless, they must be kept reasonably cool, as their internal resistance $\left(R_{D S}(\mathrm{ON})\right)$ rises with temperature. It is experimentally shown that this approach works well at relatively low-supply DC voltage $(+24 \mathrm{~V})$, providing both high output power and high efficiency. Typical WPT systems used in EV charging that match the present system's efficiency work at higher DC supply voltages (50 up to $300 \mathrm{~V}$ ).

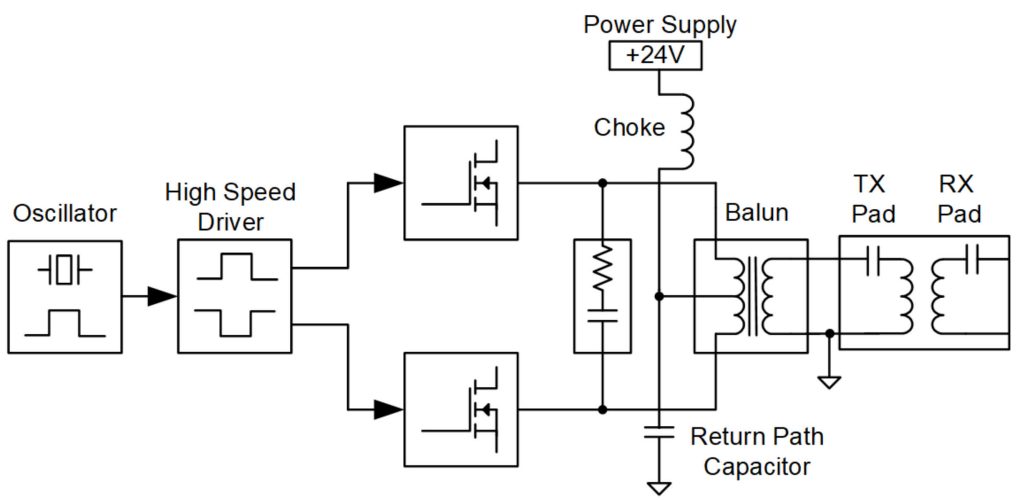

Figure 10. Block schematic of the proposed DC-AC inverter. The inverter is supplied via the balun transformer. ON/OFF switching generates a $50 \mathrm{kHz}, 48 \mathrm{~V}$ square wave in the primary windings.

The Class-E amplifiers are driven by the high-speed driver MCP14E9 through parallel resistor/diode networks which slow the gate turn ON and speed up the gate turn OFF. This provides additional OFF time between the phase transitions, helps prevent crossconduction, and provides some isolation between the two gates of the transistors that are 
placed in parallel. The transistors used in the present prototype are IRFP4227 MOSFETs with a nominal $25 \mathrm{~m} \Omega$ rds-on resistance. The high-voltage transistors were used for safety and reliability purposes, at the cost of a lower efficiency. The Zobel configuration connected between the two channels plays a key role in eliminating ringing effects and high-voltage peaks that appear on the MOSFET transistors, caused by switching transients, parasitic resonances, and reflected waves. Simulations performed with a Spice simulator were carried out to ensure that no voltages throughout the entire system surpassed $800 \mathrm{~V}$, which represents the breakdown voltage of the wire isolation used. The balun uses two $77 \mathrm{Mn}-\mathrm{Zn}$ ferrite toroids from Fair-Rite, with dimensions of $73 \times 38 \times 24 \mathrm{~mm}$, and was designed for optimal power transfer.

Single-ended Class-E amplifiers have a return current capacitor connected between the Drain and Source of each MOSFET. In the present design, the Drain-Source capacitors are only used to filter ringing effects, together with the Zobel network, while the return path capacitor is placed between the power supply's choke inductor and ground, right before the balun, as visible in Figure 10. Using the same return capacitor for both the high-side and low-side results in a better balancing, but will slightly decrease the system efficiency due to the additional copper and core losses in the balun. Since magnetic field distribution is a priority in the design of the $T x$ and $R x$ coils, the electric impedance matching must be performed by tuning the Zobel network and turning ratio of the balun.

As mentioned, the high efficiency of the Class-E amplifier is due to the fact that when the MOSFETs are OFF, the theoretical conduction current is 0 , and when the MOSFETs are $\mathrm{ON}$, the theoretical voltage is 0 . The zero-voltage switching (ZVS) and zero-voltage derivative switching (ZVDS) conditions for the proposed design are obtained by solving:

$$
\begin{gathered}
\left.v_{H}(\omega t)\right|_{\omega t=2 k \pi}=0 \\
\left.\frac{d}{d t} v_{H}(\omega t)\right|_{\omega t=2 k \pi}=0 \\
\left.v_{L}\left(\omega\left(t-\frac{T}{2}\right)\right)\right|_{\omega\left(t-\frac{T}{2}\right)=2 k \pi}=0 \\
\left.\frac{d}{d t} v_{L}\left(\omega\left(t-\frac{T}{2}\right)\right)\right|_{\omega\left(t-\frac{T}{2}\right)=2 k \pi}=0
\end{gathered}
$$

where $T$ is the driving pulse repetition interval, $\omega=\frac{2 \pi}{T}, v_{H}$ is the high-side voltage, and $v_{L}$ is the low-side voltage. Figure 11a shows the simulated results of the waveforms in the drain of the MOSFET transistors from the high-side Class-E amplifier. The low-side channel waveforms are identical, but time-shifted by 180 degrees. In Figure 11b, simulated waveforms are shown for the signals obtained at the balun transformer secondary windings and the $1 \Omega$ resistive load respectively, when the system is operating at $50 \mathrm{kHz}$. The signal fed into the $T x$ pad and the signal at the resistive load are out of phase by 90 degrees, which is consistent with the results obtained in Equations (3) and (4). By varying the number of turns in the secondary winding, the system can be tuned for better efficiency or for maximum power transfer, thus having the possibility to obtain an optimal compromise. The Class-E amplifiers were placed directly on a heavy aluminum heat sink for maximum heat dissipation. The control and protection system were placed on a FR-4 PCB at $1 \mathrm{~cm}$ above the heat sink. An RF choke and the large electrolytic capacitor are used to filter voltage and current ripples on the supply line. 


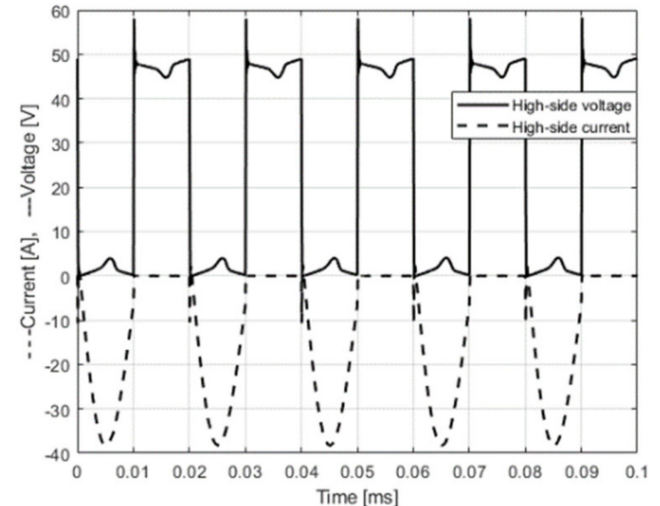

(a)

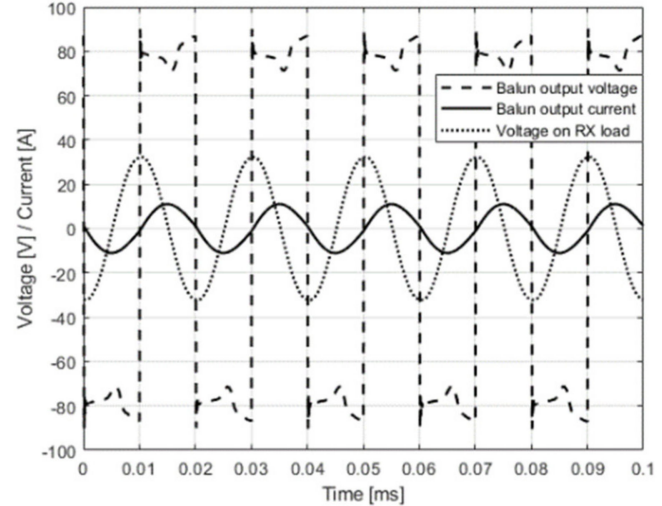

(b)

Figure 11. (a) Simulated results of the waveforms in the drain of the MOSFET transistors. The current is represented with a dashed line, and the voltage with a continuous line. (b) Simulated results of the input waveforms from the secondary winding of the balun $\mathrm{T} 1$ and the output voltage at the resistive load (RL). The voltage is represented with the dashed line, the current is represented with the continuous line, and the voltage received at the resistive load is represented with the dotted line.

\section{Experimental Results}

In this section, we first present the influence of the load impedance and positioning on the output power and the efficiency of the prototype. Load impedance changes are mainly caused by two factors: positioning errors of the UAV and the different charging stages of a Li-ion battery [32]. For the present prototype, shown in Figure 12, two series-connected $12 \mathrm{~V} / 18 \mathrm{Ah}$ lead-acid batteries were used to power the system in order to minimize supply broom noise and switching noises in the measurements. In all experiments, the load at the receiver's end was dissipative and was made out of high-power resistors. Changing the configuration in which the resistors were placed made varying the load impedance very easy and precise. When transferring maximum power, the DC supply voltage dropped to about $22 \mathrm{~V}$. The overall balancing of the power transfer and efficiency can be performed considering three factors: (a) the load impedance, (b) the coupling factor, which is provided by the distance between the $T x$ and $R x$ coils, and (c) the turn ratio of the balun transformer.

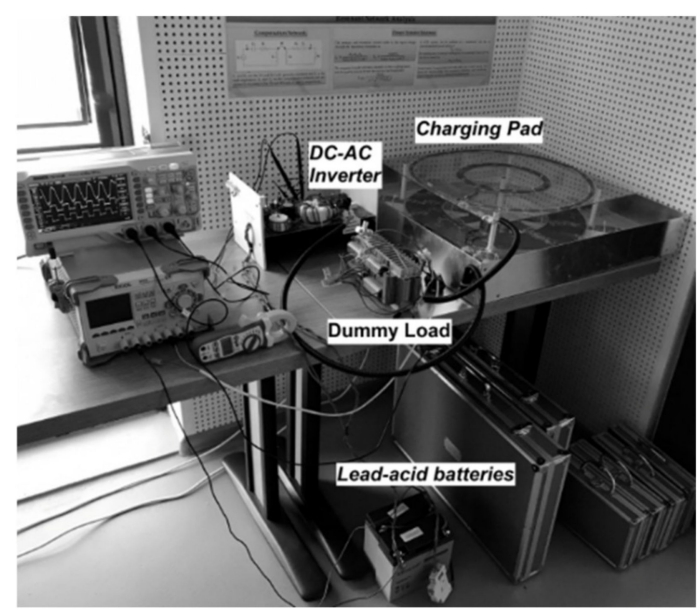

Figure 12. The proposed WPT system.

Since for a given UAV the charging distance is generally imposed, this leaves two degrees of freedom which can be used to optimize efficiency. The maximum system efficiency obtained is approximately $95 \%$, which corresponds to an output power of $625 \mathrm{~W}$ and an input power of $660 \mathrm{~W}$. The result was obtained when the $R x$ coil was placed $10 \mathrm{~cm}$ above the $T x$ pad and the load was set to $1 \Omega$. System efficiency is calculated as the ratio 
between the input DC power and the output AC power. The final AC-DC converter block is not taken into consideration since it is not within the scope of this paper, but a compatible design example can be found in [33]. The input power of the DC-AC inverter was calculated as the DC battery voltage multiplied by the current, both measured with a true RMS clamp meter, and by measuring the voltage across a high-power shunt resistor. The output power of the system was calculated as the squared RMS voltage drop on the load divided by the load's impedance. The voltage waveform obtained at the load was a $25 V_{R M S}$ sinusoid \pm 90 degrees out-of-phase, relative to the driving square wave voltage, as depicted in Figure 13. The result is consistent with both theory and the Spice simulations. The distortion seen in the load voltage waveform in Figure 13 is caused by the additional inductance of the wiring used to connect the load resistors together. Our tests and simulations have shown that this distortion was small and had a negligible effect on system efficiency.

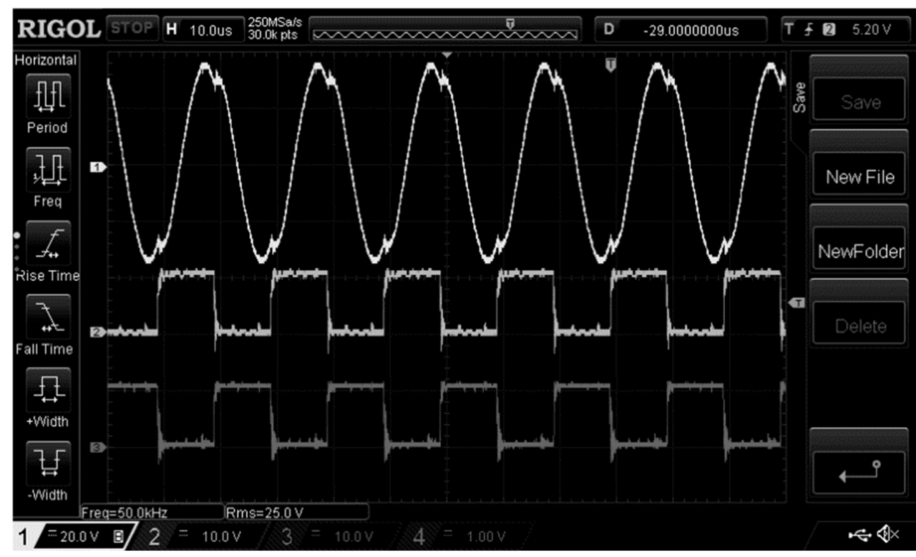

Figure 13. Waveform 1 (top) represents the output voltage across the $1 \Omega$ load, and waveforms 2 and 3 (bottom) represent the driving signals of the Class-E inverter. The $25 V_{R M S}$ and $50 \mathrm{kHz}$ readings are from waveform 1.

The efficiency and input/output power that were obtained are presented in Figure 14a,b respectively, when the load impedance was varied between 0.5 and $2.5 \Omega$. Comparing the obtained system efficiency results shown in Figure 14a and the simulated transfer efficiency from Figure 3, it can be inferred that the PA reached a peak efficiency of $98 \%$.

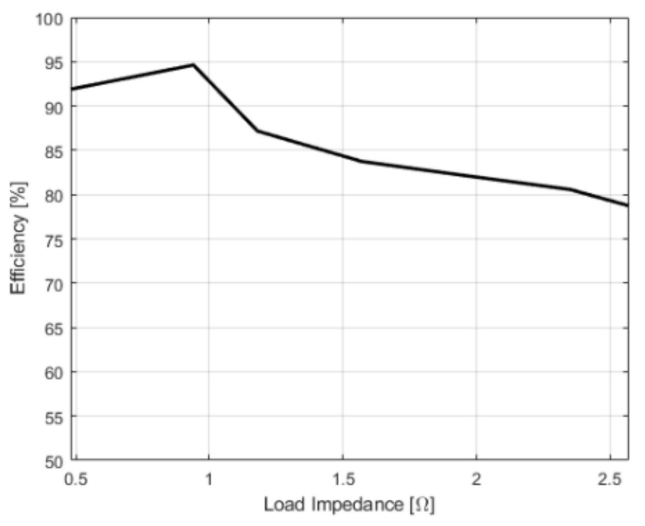

(a)

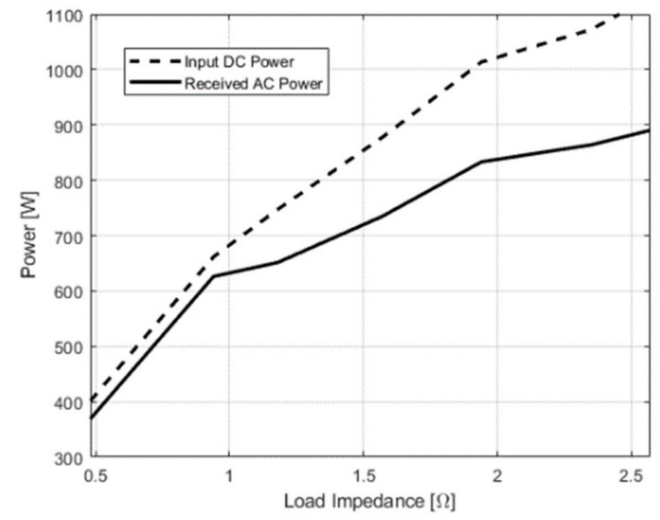

(b)

Figure 14. (a) Measured DC to AC system efficiency as a function of load impedance, for a distance of $10 \mathrm{~cm}$ between the $R x$ and $T x$, with a coupling factor of 0.29. (b) Measured input DC power (dashed line) and output AC power (continuous line).

When increasing the vertical distance between the $T x$ and $R x$ coils up to a certain point of approximately $20 \mathrm{~cm}$, the transferred power will continue to increase. This can be 
explained by revising the analysis performed in Section 2.2., more precisely Equations (3) and (4), which state that if the system is weakly coupled, the Tx current increases, giving rise to large magnetic fields. Evidently, this increased power transfer comes at the cost of a lower efficiency. The obtained experimental results are shown in Table 2.

Table 2. Vertical offset measurements.

\begin{tabular}{ccccc}
\hline Height $(\mathbf{c m})$ & Coupling & $T x$ Power $(W)$ & $R x$ Power $(W)$ & Efficiency (\%) \\
\hline 10 & 0.29 & 660 & 625 & 94.7 \\
\hline 20 & 0.11 & 1840 & 1142 & 62 \\
\hline
\end{tabular}

A similar result was achieved for horizontal offset, caused by positioning errors of the UAV. Figure 15a,b graphically present the efficiency and the input/output power respectively, as a function of horizontal positioning offset between the two center points of the $T x$ pad and the $R x$ pad. The magnetic coupling between the $T x$ and $R x$ coils is presented in Figure 16. All horizontal offset measurements were performed at a constant height of $10 \mathrm{~cm}$. By comparing the results in Table 2 and Figure 16, it can be seen that the coupling is much less sensitive to horizontal offsets than it is to vertical offsets. This is mainly due to the wide and flat horizontal field distribution of the Tx pad. Moreover, when Equation (8) is satisfied, and when the copper and core losses are very small, variations in coupling below a certain threshold have little effect on efficiency.

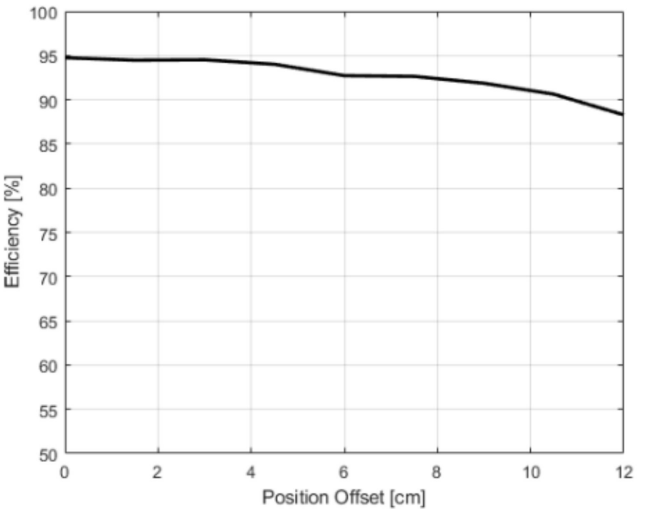

(a)

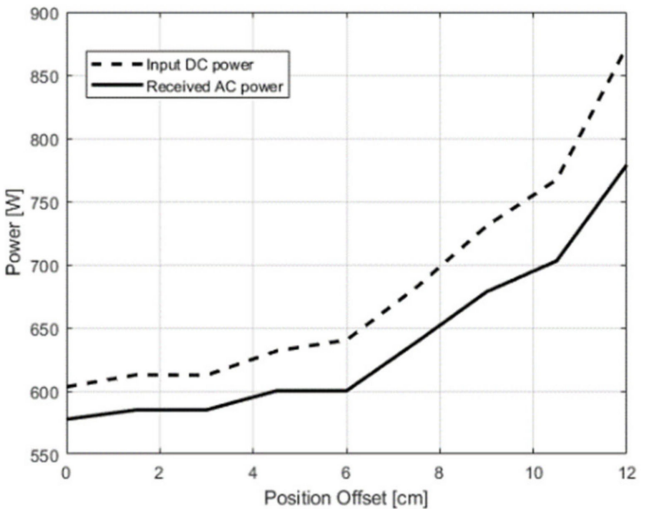

(b)

Figure 15. (a) Measured DC to AC system efficiency as a function of horizontal displacement, for a load impedance of $1 \Omega$. (b) Measured input DC power (dashed line) and output AC power (continuous line).

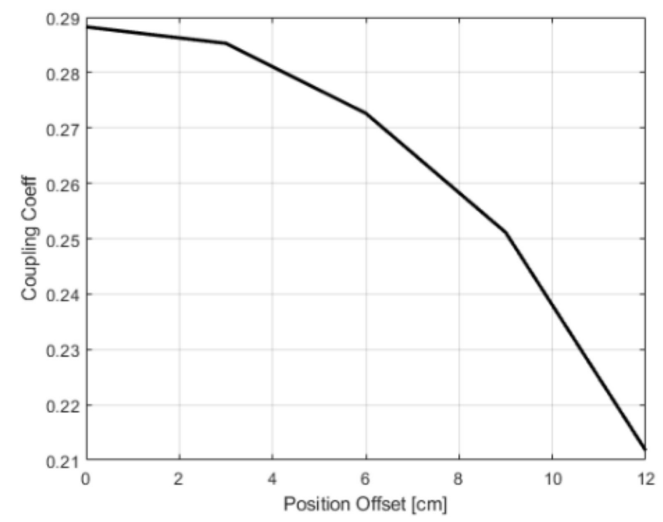

Figure 16. Measured coupling coefficient between $T x$ and $R x$ coils as a function of horizontal position offset. 


\section{Conclusions}

In this paper, a highly efficient VLF WPT charging system used for fast-charging large UAVs was presented. The proposed DC-AC inverter is based on a modified Class-E amplifier. The advantages of this solution are summarized in Table 3.

Table 3. Prior state-of-the-art system comparison.

\begin{tabular}{|c|c|c|c|c|c|c|c|}
\hline Ref. & $\begin{array}{c}\text { Efficiency } \\
(\%)\end{array}$ & $\begin{array}{c}\text { Output } \\
\text { Power }(W)\end{array}$ & $\begin{array}{l}\text { Frequency } \\
(\mathbf{k H z})\end{array}$ & $\begin{array}{c}\text { Supply } \\
\text { Voltage (V) }\end{array}$ & $\begin{array}{c}\text { Inverter } \\
\text { Configuration }\end{array}$ & $\begin{array}{c}\text { Charging } \\
\text { Distance }(\mathrm{cm})\end{array}$ & Application \\
\hline [12] & 95 & 900 & 85 & +360 & H-Brdige & 15 & EV \\
\hline [32] & 96.1 & 6600 & 67.5 & +420 & H-Bridge & 20 & $\mathrm{EV}$ \\
\hline [26] & 95 & 5000 & 20 & +600 & H-bridge & - & $\mathrm{EV}$ \\
\hline [28] & 80 & 11.75 & 750 & +8 & Class-E PA & 1.25 & Low-Power \\
\hline Proposed & 94 & 625 & 50 & +24 & $\begin{array}{c}\text { Dual-Channel } \\
\text { Class-E PA }\end{array}$ & 10 & UAV \\
\hline
\end{tabular}

The presented design showed promising opportunities and results, from both simulations and experimental results. A new $R x$ coil geometry was proposed which was designed such that it offers a sweet spot when choosing between maximum power transfer, efficiency, weight, and receiver cost. A peak system efficiency of $95 \%$ was obtained at $10 \mathrm{~cm}$, with a power transfer of $625 \mathrm{~W}$. Compared to previously mentioned designs, the present system offers the capability of similar performance but without the need of a dual-rail supply, complex matching networks, or ferrite bars at the receiver's end. A comparison with other work was presented in Table 3. However, please note that high-power transfers (as in $[12,26,32])$ can only be achieved in EV applications that do not have weight constraints. Such solutions are impossible to implement in a light UAVs with similar efficiencies.

Given the simplistic low-voltage supply requirements and the relatively reduced weight and size, the system makes an excellent candidate for a mobile deployment system for industrial and defense UAV charging applications.

Author Contributions: The research and development of the proposed Wireless Power Transfer system for UAV applications, consisting of the DC-AC inverter and the Tx-Rx pads, F.R.; The presented prototype was optimized using mathematical derivations, Spice simulations, Numerical simulations, FEM simulations and multiple experiments, A.B.; All authors have read and agreed to the published version of the manuscript.

Funding: This work was supported by a grant of the Romanian Ministry of Research and Innovation, CCCDI-UEFISCDI, project number: "PN-III-P1-1.2-PCCDI-2017 0839/19PCCDI / 2018, within PNCDI III".

Conflicts of Interest: The authors declare no conflict of interest.

\section{References}

1. Sullivan, J.M. Evolution or revolution? The rise of UAVs. IEEE Technol. Soc. Mag. 2006, 25, 43-49. [CrossRef]

2. Qazi, S.; Siddiqui, A.S.; Wagen, A.I. UAV based real time video surveillance over 4G LTE. In Proceedings of the Conference on Open Source Systems and Technologies (ICOSST), Lahore, Pakistan, 17-19 December 2015; pp. 141-145.

3. Mahardika, M.; Nugroho, G.; Prasetyo, E.Y. UAV long range surveillance system based on BiQuad antenna for the Ground Control Station. In Proceedings of the IEEE Student Conference on Research and Development (SCOReD), Kuala Lumpur, Malaysia, 13-14 December 2016; pp. 1-5.

4. Stacy, N.J.S.; Craig, D.W.; Staromlynska, J.; Smith, R.B. The Global Hawk UAV Australian deployment: Imaging radar sensor modifications and employment for maritime surveillance. In Proceedings of the IEEE International Geoscience and Remote Sensing Symposium, Toronto, ON, Canada, 24-28 June 2002.

5. Turner, D.; Lucieer, A.; Wallace, L. Direct Georeferencing of Ultrahigh-Resolution UAV Imagery. IEEE Trans. Geosci. Remote Sens. 2014, 52, 2738-2745. [CrossRef]

6. Lort, M.; Aguasca, A.; Lopez-Martinez, C.; Marin, T.M. Initial Evaluation of SAR capabilities in UAV Multicopter Platforms. IEEE J. Sel. Top. Appl. Earth Obs. Remote Sens. 2018, 11, 127-140. [CrossRef] 
7. Sharma, V.; Kumar, R.; Rana, P.S. Self-Healing Neural Model for Stabilization against Failures over Networked UAVs. IEEE Commun. Lett. 2015, 19, 2013-2016. [CrossRef]

8. Covic, G.A.; Boys, J.T. Modern Trends in Inductive Power Transfer for Transportation Applications. IEEE J. Emerg. Elect. Top. Power Electron. 2014, 1, 28-41. [CrossRef]

9. Samad, T.; Bay, J.S.; Godbole, D. Network-Centric Systems for Military Operations in Urban Terrain: The Role of UAVs. Proc. IEEE 2007, 95, 92-107. [CrossRef]

10. Li, K.; Voicu, R.C.; Kanhere, S.S.; Ni, W.; Tovar, E. Energy Efficient Legitimate Wireless Surveillance of UAV Communications. IEEE Trans. Veh. Technol. 2019, 68, 2283-2293. [CrossRef]

11. Rosu, F.; Badescu, A.; Rusu, B. Analysis and Optimization of an IPT System. In Proceedings of the SPIE Conference ATOM-N Constanta, Romania, 23-26 August 2018.

12. Lu, F.; Zhang, H.; Kan, T.; Hoffmann, H.; Mei, Y.; Cai, L.; Mi, C. A high efficiency and compact inductive power transfer system compatible with both $3.3 \mathrm{~kW}$ and $7.7 \mathrm{~kW}$ receivers. In Proceedings of the IEEE Applied Power Electronics Conference and Exposition (APEC), Tampa, FL, USA, 26-30 March 2017; pp. 3669-3673.

13. Covic, G.A.; Boys, J.T. Inductive Power Transfer. Proc. IEEE 2013, 101, 1276-1289. [CrossRef]

14. Ohira, T. What in the world is Q? IEEE Microw. Mag. 2016, 17, 42-49. [CrossRef]

15. Musavi, F; Eberle, W. Overview of wireless power transfer technologies for electric vehicle battery charging. IET Power Electron. 2014, 7, 60-66. [CrossRef]

16. Poon, A.S.Y. A General Solution to Wireless Power Transfer between Two Circular Loop. Prog. Electromagn. Res. 2014, 148, 171-182. [CrossRef]

17. Aditya, K.; Williamson, S.S. Comparative study of series-series and series-parallel topology for long track EV charging application. In Proceedings of the IEEE Transportation Electrification Conference and Expo (ITEC), Dearborn, MI, USA, 15-18 June 2014; pp. $1-5$.

18. Sallan, J.; Villa, J.L.; Llombart, A.; Sanz, J.F. Optimal Design of ICPT Systems Applied to Electric Vehicle Battery Charge. IEEE Trans. Ind. Electron. 2009, 56, 2140-2149. [CrossRef]

19. ARPANSA. Maximum Exposure Levels to Radiofrequency Fields-3 kHz to $300 \mathrm{GHz}$; Australian Radiation Protection and Nuclear Safety Agency: Melbourne, Australia, 2002.

20. Budhia, M.; Covic, G.A.; Boys, J.T. Design and optimization of Circular Magnetic Structures for Lumped Inductive Power Transfer Systems. IEEE Trans. Power Electron. 2011, 26, 3096-3108. [CrossRef]

21. Rosu, F.; Badescu, A. Patent Pending, A/01188, Submitted in 2019. Available online: https://worldwide.espacenet.com/patent/ search/family/068293301/publication/RO133690A0?q=Filip\%20Rosu (accessed on 8 September 2021).

22. Wheeler, H.A. Simple Inductance Formulas for Radio Coils. Proc. Inst. Radio Eng. 1928, 16, 1398-1400. [CrossRef]

23. Sampath, J.P.K.; Alphones, A.; Vilathgamuwa, D.M. Coil optimization against misalignment for wireless power transfer. In Proceedings of the IEEE 2nd Annual Southern Power Electronics Conference (SPEC), Auckland, New Zealand, 5-8 December 2016; pp. 1-5.

24. Hirayama, K.; Suetsugu, T.; Kurokawa, F. DC-DC converter based on voltage dividing class E amplifier. In Proceedings of the IEEE International Telecommunications Energy Conference, Broadbeach, Australia, 22-26 October 2017; pp. 535-540.

25. Hao, H.; Covic, G.A.; Boys, J.T. A Parallel Topology for Inductive Power Transfer Power Supplies. IEEE Trans. Power Electron. 2014, 29, 1140-1151. [CrossRef]

26. Chen, L.; Nagendra, G.R.; Boys, J.T.; Covic, G.A. Double-Coupled Systems for IPT Roadway Applications. IEEE J. Emerg. Sel. Top. Power Electron. 2015, 3, 37-49. [CrossRef]

27. Tang, L.-C.; Jeng, S.-L.; Chang, E.-Y.; Chieng, W.-H. Variable-Frequency Pulse Width Modulation Circuits for Resonant Wireless Power Transfer. Energies 2021, 14, 3656. [CrossRef]

28. Aldhaher, S.; Chi-Kwong Luk, P.; Bati, A.; Whildborne, J.F. Wireless Power Transfer Using Class E Inverter with Saturable DC-Feed Inductor. IEEE Trans. Ind. Appl. 2014, 50, 2710-2718. [CrossRef]

29. Wang, Q.; Che, W.; Dionigi, M.; Mastri, F.; Mongiardo, M.; Monti, G. Gains Maximization via Impedance Matching Networks for Wireless Power Transfer. Prog. Electromagn. Res. 2019, 164, 135-153. [CrossRef]

30. Borage, M.; Tiwari, S.; Kotaiah, S. Analysis and design of an LCL-T resonant converter as a constant-current power supply. IEEE Trans. Ind. Electron. 2005, 52, 1547-1554. [CrossRef]

31. Pantic, Z.; Sanzhong, B.; Lukic, S. ZCS LCC-compensated resonant inverter for inductive-power-transfer application. IEEE Trans. Ind. Electron. 2011, 58, 3500-3510. [CrossRef]

32. Vu, V.B.; Tran, D.H.; Choi, W. Implementation of the Constant Current and Constant Voltage Charge of Inductive Power Transfer Systems with the Double-SidedLCCCompensation Topology for Electric Vehicle Battery Charge Applications. IEEE Trans. Power Electron. 2018, 33, 7398-7410. [CrossRef]

33. Tashakor, N.; Khooban, M. An Interleaved Bi-directional AC-DC Converter with Reduced Switches and Reactive Power Control. IEEE Trans. Circuits Syst. II Express Briefs 2020, 67, 132-136. [CrossRef] 